\title{
Superburst Oscillations: ocean and crustal modes excited by X-Ray bursts
}

\author{
Frank R. N. Chambers and Anna L. Watts \\ Anton Pannekoek Institute for Astronomy, University of Amsterdam, Postbus 94249, 1090 GE \\ Amsterdam, The Netherlands \\ email: f.r.n.chambers@uva.nl
}

\begin{abstract}
Superburst oscillations are high frequency X-ray variations observed during hours' long superbursts on accreting neutron stars. We investigate a potential mechanism to explain these observations; a buoyant r-mode, excited in the ocean layers of the star. These modes are affected by ash composition in the ocean so are a good probe of nuclear burning processes. The phenomenon could be used in pulse profile modelling as a way of measuring neutron star mass and radius, and so the dense matter equation of state.
\end{abstract}

Keywords. stars: neutron, X-rays: bursts, stars: oscillations, X-rays: binaries

\section{Introduction}

Superbursts are hours' long X-ray bursts that are observed from accreting neutron stars. They are believed to be caused by unstable thermonuclear carbon burning deep in the ocean, and are different to ordinary H/He triggered X-Ray bursts which last seconds to minutes, in which fuel is burned higher up in the atmosphere and caused by hydrogen or helium burning.

Burst oscillations are high frequency variations in X-ray luminosity (Watts 2012) and were observed during $\mathrm{H} / \mathrm{He}$ triggered bursts in $4 \mathrm{U}$ 1636-536 (Muno, et al. 2002), at $580 \mathrm{~Hz}$ and drift by $2 \mathrm{~Hz}$ as the as burst cools. They have also been observed during a superburst in the same source (Strohmayer \& Markwardt 2002), $2 \mathrm{~Hz}$ higher and more stable than those observed in $\mathrm{H} / \mathrm{He}$ triggered burst, drifting by an amount expected due to orbital Doppler shift. Two main models have been put forward to explain burst oscillations for $\mathrm{H} / \mathrm{He}$ triggered bursts; flame-spread-halting and global-modes. The mode model is attractive since it explains the frequency drifts in the $\mathrm{H} / \mathrm{He}$ triggered bursts, but no model fits perfectly.

\section{Results}

There are many different mode candidates, but the buoyant r-mode with low $m$ still remains one of the most promising, at least for $\mathrm{H} / \mathrm{He}$ triggered bursts (Heyl 2004). We present a calculation of the frequency evolution of this mode during a superburst using a similar approach to that used by Piro \& Bildsten (2005) for H/He triggered bursts, which has never been done for superburst oscillations. Modes are found by perturbing a static cooling background, assuming a plane parallel atmosphere, adiabatic perturbations, and crust coupling. The depth of thermonuclear burning is different for the two cases, at column depth $3 \times 10^{8}, 10^{12} \mathrm{~g} \mathrm{~cm}^{-2}$ for the $\mathrm{H} / \mathrm{He}$ triggered burst and superburst respectively. 


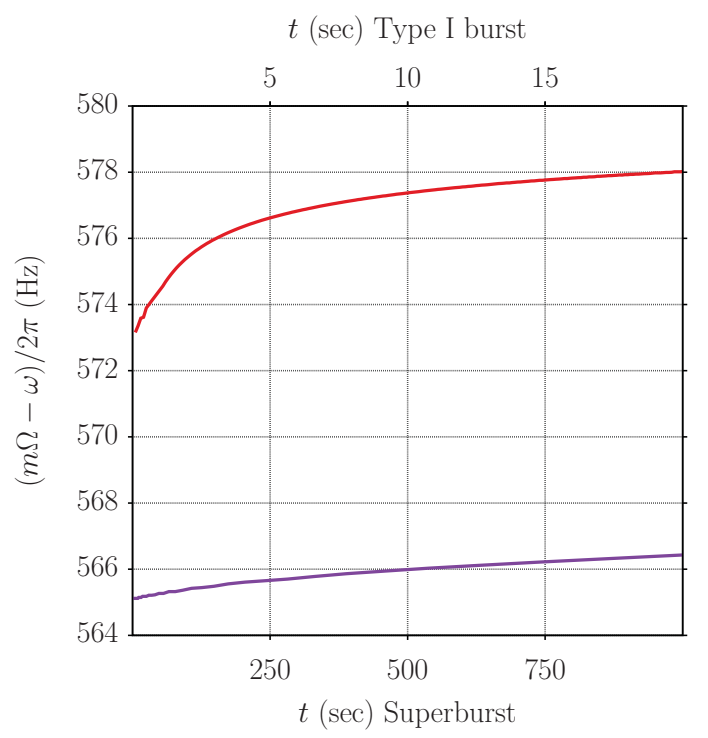

Figure 1. Frequency in the inertial frame of buoyant $m=1 \mathrm{r}$-mode evolving throughout: superburst (lower line) and $\mathrm{H} / \mathrm{He}$ triggered X-ray burst (higher line). Frequency drift is caused by cooling after a burst. For the superburst we show the first 1000 seconds, this is the time over which the oscillations were observed in the $4 \mathrm{U} 1636-536$ superburst. The spin frequency of the star is $582 \mathrm{~Hz}$

The model of (Cumming \& MacBeth 2004) is used to calculate the stationary background during the burst, it is non-relativistic, assumes constant gravity, hydrostatic equilibrium, and evolves due to radiative and neutrino cooling. Initial conditions are a hot layer of mixed Carbon and Iron $\left(X_{C}=0.3\right)$ on top of a cooler layer of pure Iron.

As can be seen from Figure 1, the mode does not match observations. For $\mathrm{H} / \mathrm{He}$ triggered bursts, the model predicts frequency drifts of $9 \mathrm{~Hz}$, greater than the $2 \mathrm{~Hz}$ drift in the observations. This is a known problem and not yet solved (Piro \& Bildsten 2005; Berkhout \& Levin 2008). The superburst model predicts a frequency drift of 2 $\mathrm{Hz}$, whereas the observed superburst oscillations exhibit no drift beyond that expected due to orbital Doppler shifts over the $\sim 1000$ s that they are detectable. In addition, the observed superburst oscillation frequency is above that for the $\mathrm{H} / \mathrm{He}$ triggered burst. The buoyant r-mode frequency calculated in our model, on the other hand, is lower. In its simplest form, the buoyant r-mode cannot explain the superburst oscillations. We are now developing the model further (Chambers et al. in prep.).

\section{Acknowledgement}

ERC Starting Grant No. 639217 CSINEUTRONSTAR (PI Watts)

\section{References}

Berkhout, R. G., Levin, T., 2008 MNRAS. 385:10291035

Cumming, A., MacBeth, J., 2004 ApJ. Lett. 603:L37L40

Chambers, F. R. N., Watts, A. L., Cavecchi, Y., Garcia, F., Keek L., in prep.

\& Heyl, J. S., 2004, ApJ 600:939945

Muno M. P. Özel, F., Chakrabarty D. 2002, ApJ. 581:550561

Piro, A. L., L. Bildsten, L., 2005 ApJ. 629:438450

Strohmayer, T. E., Markwardt, C. B., 2002 ApJ, 577:337345

Watts, A. L., $2012 A R A \& A$, 50:609640, 2012. 